\title{
Criterios de diseño para las edificaciones educativas del estado Zulia, Venezuela
}

\author{
Design criteria for educational buildings in the state of Zulia, Venezuela
}

Raymundo Portillo Ríos, ${ }^{1}$ M.Sc.

${ }^{1}$ Universidad del Zulia, Facultad de Arquitectura y Diseño, Zulia, Venezuela. Correspondencia: raymundo.portillo@fad.luz.edu.ve

Recibido: 2 de mayo 2016. Aceptado: 23de julio 2016.

Portillo, R. (2016) Criterios de diseño para las edificaciones educativas del estado Zulia, Venezuela. Procesos urbanos Número 3, Ene-Dic. 120-132. Doi:10.21892/2422085X.272

\section{RESUMEN}

Este trabajo busca determinar los criterios de diseño para las edificaciones de las unidades educativas públicas del estado Zulia. Para ello, se planteó una investigación holística, descriptiva con enfoque cualitativo, con diseño descriptivo documental y multi-variable de rasgo (eventual). Las instituciones educativas públicas agrupan la mayor cantidad de niños y jóvenes inscritos en el sistema escolar venezolano, siendo el estado Zulia la región con la mayor cantidad de matrícula escolar. De tal manera, que los principales beneficiarios de este estudio son los organismos encargados de ejecutar las políticas públicas en términos de infraestructura escolar, así como también las comunidades servidas con dichos espacios. El procedimiento de análisis fue cualitativo, con la intención de categorizar los datos de las fuentes utilizadas. Los mismos se hicieron siguiendo los aspectos formales, espaciales, funcionales, ambientales y la técnica constructiva de las edificaciones estudiadas. Desde esta perspectiva, se enumeran los criterios generales de diseño para las edificaciones educativas públicas de la región, enmarcados en la normativa vigente de FEDE.

Palabras clave: Criterios de diseño, edificaciones educativas, arquitectura escolar, escuelas públicas

\begin{abstract}
This work seeks to determine the design criteria for the buildings of the public educational units of the state of Zulia. For this, a holistic, descriptive research was proposed with a qualitative approach, with descriptive documentary design and multi-variable trait (eventual). Public educational institutions bring together the largest number of children and young people enrolled in the Venezuelan school system, with Zulia being the region with the highest school enrollment. Thus, the main beneficiaries of this study are the agencies responsible for implementing public policies in terms of school infrastructure, as well as the communities served with such spaces. The analysis procedure was qualitative, with the intention of categorizing the data of the sources used. They were made following the formal, spatial, functional, environmental aspects and the constructive technique of the buildings studied. From this perspective, the general design criteria for the public educational buildings of the region, framed in the current FEDE regulations, are listed.
\end{abstract}

Keywords: Design criteria, educational buildings, school architecture, public schools

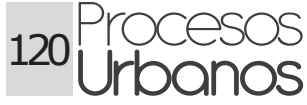


Portillo, R. - Criterios de diseño para las edificaciones educativas del estado Zulia, Venezuela.

\section{INTRODUCCIÓN.}

La educación es el proceso integral de formación de la persona, a través de métodos e instrumentos, que dotan a la misma de valores y conocimientos a fin de que esta pueda desarrollarse en cualquier área humana; de allí la importancia que tiene para cualquier sociedad invertir recursos para garantizar a su población, óptimos estándares de calidad en lo relativo a la enseñanza y la educación. En esta línea la Organización de la Naciones Unidas (ONU), planteó entre los objetivos del milenio para el año 2000, el principio: "La educación para todos", en el que 164 naciones del mundo se comprometieron a realizar esfuerzos, a fin de alcanzar en los primeros decenios del siglo XXI, que la población culminara el ciclo de educación primaria (UNESCO, 2015).

Entre los países que se comprometieron a realizar este esfuerzo mancomunado en pro de su población, estuvo la República Bolivariana de Venezuela, la que después de muchos años de debates y consultas, logró conceptualizar un nuevo marco legal, que rigiera las actividades educativas en la nación, concretada dicha propuesta en la Ley Orgánica de Educación, sancionada y aprobada por la Asamblea Nacional en el año 2009; la misma señala que la educación es un derecho humano y un deber social fundamental, orientada al desarrollo del potencial creativo de cada ser humano en condiciones históricamente particulares, razón por la cual constituye el "eje central en la creación, transmisión y reproducción de las diversas manifestaciones y valores culturales, invenciones, expresiones, representaciones y características propias para apreciar, asumir y transformar la realidad" (Asamblea Nacional, 2009; art. 4). Una de las particularidades de la Ley Orgánica de Educación del 2009, fue catalogarla función del Estado como docente y responsable principal de la actividad educativa en el país, teniendo entre muchas otras funciones la tarea de regular, controlar y supervisar "la calidad de la infraestructura educativa oficial y privada de acuerdo con los parámetros de uso y diseño" (Asamblea Nacional, 2009; art. 6, núm. 2d). Se mantiene de esta manera la tradición de concebir al Estado como el principal financista, proyectista y constructor de la infraestructura educativa nacional.

Con tales fines se fundó en 1977 la Fundación de Edificaciones y Dotaciones Educativas (FEDE), ente gubernamental dedicado "a dar respuestas a la problemática de la planta física educativa a escala nacional, atendiendo de forma sistemática las etapas correspondientes a la construcción, dotación, rehabilitación y mantenimiento del conjunto escolar" (FEDE, 2015). El mismo, con el paso del tiempo, ha sido el órgano regente en cuanto a políticas de infraestructura educativa, en todas sus dimensiones, tanto para la proyección y construcción de nuevas escuelas, así como también de la rehabilitación, mantenimiento, dotación y recuperación de las ya existentes.

Según datos del Ministerio del Poder Popular para la Educación, en Venezuela existe un déficit de más de 3.000 escuelas, entre las que se agrupan: pre-escolares, escuelas básicas, simoncitos (pres-escolares con guarderías) y liceos para la educación secundaria, siendo esta, junto con la Gran Misión Vivienda, una de las prioridades en materias de infraestructura del ejecutivo nacional, el cual afirmó que para el periodo escolar 2014-2015, se ejecutaron 150 escuelas en el territorio nacional, quedando pendientes en vía de construcción y con presupuesto aprobado más de doscientas unidades escolares (Ministerio del Poder Popular para la Educación, 2014).

Otro aporte interesante del tema fue el presentado por López et al. (2010) donde afirma que el $70 \%$ de los planteles escolares existentes en Venezuela, están en áreas de elevada amenaza sísmica, lo que representa una condición particular al momento de entender el riesgo que implica enfrentar cualquier movimiento telúrico. El trabajo de los autores señala además que aproximadamente el $46 \%$ de planteles identificados para el proyecto de investigación realizado, fueron construidos con normas antiguas que de ninguna manera satisfacen los requerimientos sismo resistentes exigidos en las normas vigentes. Según dicha investigación, en Venezuela existen 
más de 14 tipologías escolares, realizadas en los últimos 70 años, cada uno de estos modelos se adapta al contexto particular, y se repite con poca o ninguna diferencia a pesar de la relatividad que puedan presentar las diferentes zonas del país.

Más específicamente en el estado Zulia, el 30 de diciembre de 2002, y bajo decreto publicado en la Gaceta Oficial ordinaria del estado Zulia no. 4851, se crea la Fundación para la Infraestructura Educativa del estado Zulia, FUNDAEDUCA, siendo este el organismo del ejecutivo regional, para aplicar y ejecutar las políticas públicas en materia de infraestructura educativa, a lo largo y ancho de toda la geografía zuliana, surgiendo esta como fruto de la experticia técnica de la coordinación regional UCER-Z, la cual, hasta ese momento, se ocupó de la dirección del mejoramiento, fortalecimiento, consolidación de los programas de rehabilitación y dotación de las entidades educativas de la región zuliana (Gobernación del estado Zulia, 2002).

Aunque FUNDAEDUCA surge como un órgano de gobierno autónomo, y con independencia financiera, adscrito a la Secretaria de Educación del gobierno regional del estado Zulia, este debe seguir lo establecido por las normas y criterios para la infraestructura escolar, emanadas por FEDES, entidad con competencia nacional, y encargada de diseñar y regir el Plan Nacional de Construcción, Ampliación, Dotación y Mantenimiento de la Planta Física Educativa; "en los niveles de educación preescolar, básica, media y diversificada, así como también en las modalidades de educación especial, para luego someterlo a la aprobación del Ministerio del Poder Popular para la Educación" (FEDE, 2015).

En el estado Zulia, y siguiendo los criterios de diseño implantados por FEDE, las edificaciones educativas de básica y presescolar se componen por módulos tipos (aulas, oficinas, salas sanitarias, entre otros) que se organizan siguiendo diversos aspectos, entre los que se cuentan: la necesidad suministrada por la matrícula escolar, las condiciones de la parcela o terreno donde se ejecuta la obra, así como ${ }^{122}$ Urocesos también, el presupuesto asignado de lo cual depende la ejecución en años de la escuela. Según datos del Instituto Nacional de Estadísticas, en el estado Zulia, para el período lectivo 2012-2013, existía una matrícula de casi quinientos mil estudiantes, inscritos en las diversas modalidades, convirtiéndose en el estado con mayor número de estudiantes, exceptuando la zona central del país. Resultando entonces, que entre las dependencias públicas las más utilizadas se hallan en el estado Zulia, lo que hace destacar la importancia que tienen las políticas en materia de infraestructura educativa, ya que en estas instituciones atienden el mayor número de estudiantes de la región, por esta razón se escoge dicha zona para realizar el presente estudio.

\section{METODOLOGÍA.}

El objetivo general planteado en esta investigación fue determinar los criterios de diseño para las edificaciones de las unidades educativas públicas del estado Zulia. Para ello se hizo una revisión documental de las normas FEDE, así como también de la reflexión teórica de diversos autores, a fin de proponer criterios de diseño para las edificaciones educativas de básica y pre - escolar. Enmarcado en la perspectiva holística, el tipo de investigación fue descriptivo, aplicando un diseño documental, el cual consistió en describir un evento o situación y cuya fuente de datos está constituida por documentos.

La población estuvo conformada por FUNDAEDUCA, la cual está suscrita a la Secretaría de Educación, ente del gobierno regional, y las unidades de estudio o fuentes de información fueron aquellos elementos que componen los proyectos arquitectónicos de las unidades educativas públicas locales. El procedimiento de análisis fue cualitativo no estadístico, con la intención de categorizar los datos de las fuentes utilizadas.

\section{CRITERIOS DE DISEÑO.}

Fonseca (1994:9) afirma que, para el diseño arquitectónico es necesario desarrollar un proceso complejo, en donde intervienen diferentes elementos, ya que "cada género 
Portillo, R. - Criterios de diseño para las edificaciones educativas del estado Zulia, Venezuela.

de edificio posee diferentes estructuras funcionales y espaciales". Por lo que cada proyecto y proceso es diferente, en donde no existen patrones, ni recetas, sino la habilidad y experiencia que se desarrollan en las etapas del proceso para dar solución al problema de diseño arquitectónico.

De tal manera que el autor (1994:9), señala que no es posible establecer una norma para solucionar un tipo de proyecto en específico, "pues cualquier género de edificio posee características únicas en su estructura" y espacio funcional, ya que atiende a necesidades particulares. Dicha premisa descarta la aplicación de los criterios de diseño, entendidos estos de manera muy genérica como normas aplicables para el trabajo de producciones seriadas de elementos arquitectónicos, es decir, normas o propuestas que bien pueden aplicarse a varias edificaciones, que compartan los mismos usos y en las que puedan aplicar las mismas consideraciones.

Por otra parte, Rapoport (2004), entiende los criterios de diseño como el conjunto de aspectos de elección que no están bajo el control explícito de los diseñadores y que se convierten de esta manera en factores determinantes del entorno construido. Por lo cual los mismos componen un sistema de reglas útiles que dotan de características a las diferentes formas que constituyen el diseño arquitectónico.

Para el autor, los criterios de diseños condicionan enormemente la propuesta, y los mismos pueden ser tan complejos o diversos como el mismo proceso. Entre las mencionadas se encuentran: "reglas formales, explícitas, escritas, legalistas (y legalmente ejecutables) límites del terreno, distancia de la calle, superficie edificable, normativa de seguridad, salubridad, anti incendios, normas estéticas y muchas otras" condicionantes (Rapoport, 2004. P. 114).

En términos generales existen varios tipos de criterios de diseño, porque pueden abarcar desde aspectos ideológicos, culturales, religiosos, ambientales, climatológicos, legales, tecnológicos, programáticos y estéticos; los mismos son empleados a elección del diseñador, lo cual constituye un aspecto fundamental en el proceso de diseño arquitectónico, ya que la consideración de dichos criterios y el énfasis que se le dé a cada uno de ellos, generará diversos resultados o soluciones al problema abordado, afirmando de esta manera que "diferentes criterios conducen a diferentes resultados" (Rapoport, 2004. P. 115).

Para Sanoff (2006), los criterios de diseño constituyen la tercera etapa a considerar en el proceso de diseño arquitectónico, los mismos son precedidos por la revisión bibliográfica que se hace del tema a desarrollar, y por la descripción de los usuarios y/o clientes, y ellos preparan el camino para la definición del programa arquitectónico y las especificaciones espaciales a considerar durante el desarrollo del trabajo.

Los criterios de diseño según Sanoff (2006), deben por tanto identificar a los usuarios de las instalaciones, sus actividades, actitudes y características, a partir de los cuales se establezcan aspectos funcionales para las necesidades de espacio y circulación. Además deben considerar el entorno natural, la seguridad, las superficies, los acabados y la flexibilidad del diseño. En síntesis, los criterios identifican los objetos de diseño que se proponen, las opciones de programa para cada objeto y la opción que se evalúe en términos de costos-beneficios y la rentabilidad para los requerimientos de espacio (Sanoff, 2006).

Loyola y Goldsack (2010), desde una visión más práctica, asocian el concepto de criterios de diseño, a la calidad del diseño, y por ende a la materialización del mismo. Para los autores el cumplimiento de los criterios de diseño (desempeño o funcionamiento del edificio) son los que pueden garantizar la calidad del proceso constructivo, los mismos se asocian a estándares de desempeños, que deben satisfacer la necesidad espacial de los clientes y usuarios considerados para el diseño arquitectónico.

Sin embargo, los mismos no representan necesariamente "un factor de constructividad", sino que corresponden a una característica propia del proceso de 


\section{Procesos Urbanos N³ Enero - Diciembre; 2016}

diseño y de las soluciones que se generan de este; entre ellos se agrupan factores de costos económicos, el programa arquitectónico, los aspectos relativos al desempeño energético y la normativa aplicable, para cada uno de los casos (Loyola y Goldsack, 2010. P. 69).

En síntesis, los criterios de diseños son aspectos básicos "que orientan las decisiones de diseño, señalando la tendencia generalmente adecuada en la mayoría de las situaciones de diseño" que se pretenden solucionar con el edificio arquitectónico, los mimos pueden ser principios, pautas, y reglas que constituyen factores, internos, externos y transversales que determinan la materialización o construcción de la edificación (Loyola y Goldsack, 2010. P. 70).

Desde esta perspectiva, puede afirmarse que los criterios de diseño son principios, pautas y reglas genéricas, que condicionan los aspectos considerados en el proceso de diseño arquitectónico. Los mismos abarcan aspectos funcionales y espaciales, de forma y estilo, así como ambientales y técnicos constructivos; la consideración y la aplicación de cada uno de ellos, debe generar soluciones de diseño, que busquen satisfacer la necesidad espacial abordada.

\section{EDIFICACIONES EDUCATIVAS.}

La norma FEDE, define a la escuela como "el edificio educativo: construcción cubierta que alberga alguna actividad relacionada con la función educativa del plantel". Es la unidad constituida por un edificio o conjunto de edificios ubicados, en un mismo terreno o parcela en los que funciona uno o varios planteles, o bien una parte de ellos (Gerencia de proyectos FEDE, 2007. P. 16).

Gutiérrez (2009. P. 161) señala que, "los edificios escolares representan modelos de enseñanza particulares; por tanto, cuando hay un cambio en los conceptos sobre la educación, este se refleja en un nuevo tipo de diseño de los espacios". Siendo lo más importante según el autor, el modelo de educación y pedagogía que se pretenda impartir, lo cual va a acondicionar en la configuración espacial y la propuesta arquitectónica del edificio escolar.
En esta dinámica espacio-pedagogía, o bien, edificio-escuela, juegan un papel preponderante los roles de los participantes, a saber del profesor como facilitador del proceso y de los estudiantes como actores primordiales en el mismo; en este sentido, "lo que debe intentar la propuesta arquitectónica es una transformación en la cual el estudiante sea más activo, permitiendo una relación constante con el entorno" (Gutiérrez, 2009. P. 162).

El diseño de un edificio escolar, debe surgir pues de esta dinámica, en la que intervienen diversos aspectos, más que funcionales, sino más bien pedagógicos; criterios que lo inserta en el tramado de servicios urbanos en la ciudad. Por tanto: "no debe existir un contraste marcado entre el edificio escolar y la arquitectura de la ciudad. El diseño del plantel educativo debe seducir a los estudiantes e invitarlos a participar de la escolaridad, como algo integrado a las demás actividades del entorno" (Gutiérrez, 2009. P. 164).

Ahora bien, una unidad educativa está conformada por el pre-escolar y la escuela básica en sus dos etapas, entendiendo la primera como el nivel que se orienta a niños y niñas desde los tres años y un mes hasta cumplir los seis años o hasta su ingreso al primer grado de la educación básica.

"El pre-escolar ofrece atención en instituciones educativas, en las escuelas y en la comunidad, en el área de pedagogía ejecutada por distintos sectores educativos o personas significativas, que promueven experiencias de aprendizaje que faciliten el desarrollo pleno de sus potencialidades, para que puedan encarar con éxito la escolarización de la educación básica" (Gerencia de proyectos FEDE, 2007. P. 17).

Por otra parte, el nivel de educación básica es entendido como aquel que:

"Tiene como finalidad contribuir a la formación integral del educando mediante el desarrollo de sus destrezas y su capacidad científica, técnica, humanista y artística. Cumplir funciones de exploración, de orientación educativa y vocacional para 
Portillo, R. - Criterios de diseño para las edificaciones educativas del estado Zulia, Venezuela.

iniciarlos en el aprendizaje de disciplinas y técnicas que le permitan el ejercicio de una función socialmente útil; estimulando así el deseo de saber y desarrollar la capacidad de ser de cada individuo de acuerdo de sus aptitudes. Tendrá una duración no menor de seis (6) años organizados en dos (2) etapas de la siguiente forma: A la 1ra etapa le corresponde el primero, segundo y tercer grado para los niños de 7, 8 y 9 años de edad respectivamente. A la 2da etapa le corresponde el cuarto, quinto y sexto grado, para los niños de 10, 11 y 12 años de edad respectivamente" (Gerencia de proyectos FEDE, 2007 P. 17).

\section{CRITERIOS DE DISEÑO PARA LAS ESCUELAS DEL ESTADO ZULIA}

Los criterios generales de diseño para las edificaciones educativas públicas del estado Zulia, son principios, pautas y reglas genéricas, que determinan los elementos considerados en el proceso de diseño arquitectónico. Los mismos abarcan aspectos funcionales y espaciales, de forma y estilo, así como ambientales y técnicos constructivos. Se entiende que el edificio escolar es el escenario en donde se realizan actividades de formación integral para niños, niñas, jóvenes y adolescentes, tanto de pre-escolar (niños de 3 a 6 años), como de la escuela básica primaria en su dos etapas (niños y jóvenes de 6 a 12 años); las mismas son financiadas y ejecutadas por los organismos públicos de gobierno regional con competencia en esta materia.

Por lo que estos criterios se plantean, según lo indicado por Gutiérrez (2009), donde señala que, los edificios escolares representan modelos de enseñanza específicos, los cuales se reflejan en los nuevos tipos de espacios escolares diseñados.

Teniendo como base esta premisa se formulan los siguientes tipos de diseño, con base en la teoría estudiada, y a la descripción de la variable; hay que añadir además que para la presente propuesta se han considerado las normas y recomendaciones para el diseño de las edificaciones educativas, propuestas por FEDE (Gerencia de proyectos FEDE, 2007), a fin de cumplir con los reglamentos nacionales al respecto. Los criterios de diseño presentados se irán enumerando para su fácil revisión.

\section{Programación arquitectónica.}

Las normas FEDE, conciben la programación arquitectónica de las unidades educativas escolares, desde una doble perspectiva: en primer lugar señalan los espacios necesarios referidos al pre-escolar, y en segundo lugar para la escuela básica; para ello se identifican cinco áreas o sectores prioritarios: Docentes, administrativos, servicios, circulación, áreas exteriores.

Ambos programas son desarrollados individualmente de la siguiente manera:

\section{Para pre-escolar:}

Sector docente: comprendido por aulas flexibles para el desarrollo de al menos cuatro (4) áreas de actividad con facilidades para depósitos de materiales y equipos de enseñanza y con relación directa al área de sanitarios, se proponen de manera consolidada en un esquema básico.

Sector administrativo: Comprende la dirección o coordinación, los espacios de secretaría y archivo, el área de recibo y espera, la sala de profesores, y los sanitarios (profesores).

Sector servicio: compuesto por el espacio para la enfermería, el depósito general, el depósito de material docente, el vestuario de bedeles, módulo de servicios (para caseta hidroneumática e instalaciones), la cocina y el comedor.

Sector áreas exteriores: compuesto por las áreas verdes, caminerías, áreas pavimentadas y los estacionamientos.

Se propone incluir en el área de exteriores, un espacio a modo de patio de formación o plaza cívica, que sirva como espacio conector y articulador de los espacios.

\section{Para la escuela básica:}

Sector docente: compuesto por aulas, taller de artes plásticas, aula de música, salón de usos múltiples, gimnasio o cancha deportiva, aula de computación (C.B.T. Centro bolivariano de tecnología) y taller de computación. 
Sector administrativo: compuesto por la dirección, subdirección, secretaría y espera, archivo, área para el comité curricular, sala para equipo de evaluación, control de estudio y espacio para la comunidad educativa.

Sector de servicio: referido a cocina, comedor, cafetín, módulo servicio (electricidad, basura y bombas), sanitarios, vestuario para bedeles, sanitarios para estudiantes (as), y depósito general.

Sector de áreas exteriores: áreas verdes, áreas pavimentadas, estacionamentos.

Se propone incluir en el área de exteriores, un espacio a modo de patio de formación - plaza cívica, que sirva como conector y articulador de los espacios, así como también una plataforma, tarima o elevación a desnivel, que sirva para la realización de actos culturales, la misma podría considerarse según el ancho del patio y con una altura no menor a las $60 \mathrm{cms}$. lo cual considera solo cuatro escalones para su acceso.

Se recomienda mantener la conserjería dentro del sector de servicio, la experiencia en la región, hace considerar como positiva la alternativa en materia de seguridad, sobre todo en los periodos vacacionales, ya que la familia que allí reside se hace responsable del resguardo de los bienes, así como de avisar a las autoridades, ante alguna situación presentada.

\section{Para las aulas:}

Se recomiendan esquemas ortogonales, a fin de garantizar la flexibilidad y la alternabilidad del mobiliario según los diferentes usos, para ello se proponen una modulación de $7 \mathrm{mts}$. $x$ 7,5 mts. con lo cual se optimiza la norma FEDE en cuanto al metraje por estudiante; así como también debe atenderse el criterio de no ubicar aulas de prescolar en planta alta, ni los tres primeros cursos de la escuela básica. Esta premisa se fundamenta en lo señalado por Gutiérrez (2009) quien indica que la propuesta arquitectónica debe llevar a la participación activa del estudiante en el proceso educativo (Tabla 1 ).

\section{En cuanto a los accesos:}

Es recomendable mantenerlos por separado los accesos de básica y de pre-escolar, sin embargo, se recomienda añadir el de servicio y de mantenimiento y el de vehicular correspondiente a los estacionamientos. Los mismos deberán dotarse de espacios de espera externos, ya que la entrada y la salida de estudiantes puede provocar aglomeraciones de personas (estudiantes, familiares y representantes) en los accesos del plantel. "Se exige un área de espera externa junto o al lado de la entrada para personas de 5.00 metros cuadrados por aula, que no interrumpan las condiciones para el tránsito a personas discapacitadas" (Gerencia de proyectos FEDE, 2007. P 111) y (Gutiérrez, 2009).

Por lo cual se señalan cuatro accesos a definir en el diseño: - Acceso para estudiantes de básica. - Acceso para estudiantes de pre-escolar. - Acceso de servicio. - Acceso vehicular.

Según la norma FEDE, las escuelas deben tener un estacionamiento interno mínimo para profesores, director, funcionarios y visitantes. Para carga y descarga mínimo un

Tabla 1. Área por aulas según el número de estudiantes.

\begin{tabular}{|c|c|c|c|c|c|}
\hline Espacios & $\begin{array}{c}\mathrm{n}^{\circ} \mathrm{de} \\
\text { estudiantes }\end{array}$ & $\begin{array}{c}\text { m2 } x \text { estudiantes } \\
\text { FEDE }\end{array}$ & $\begin{array}{c}\text { M2 x estudiantes } \\
\text { Propuesta }\end{array}$ & $\begin{array}{c}\text { Área por aula } \\
\text { FEDE }\end{array}$ & $\begin{array}{c}\text { Área } \\
\text { por aula } \\
\text { Propuesta }\end{array}$ \\
\hline $\begin{array}{c}\text { Aula de pre- } \\
\text { escolar }\end{array}$ & 25 & 1.72 Mts. 2 & 1.75 Mts. 2 & 42 Mts. 2 & 43.75 Mts. 2 \\
\hline Aula de básica & 30 & $1.36 \mathrm{Mts} .2$ & 1.75 Mts. 2 & 40.8 Mts. 2 & 52.5 Mts. 2 \\
\hline
\end{tabular}

Fuente: elaboración propia (2016). 
Portillo, R. - Criterios de diseño para las edificaciones educativas del estado Zulia, Venezuela.

(1) puestos y uno o dos (2) puestos para discapacitados, en las zonas urbanas; este puede también tener cuando se requiera un espacio del estacionamiento para el autobús escolar cuando sea necesario. En el resto de los casos es recomendable prever una parada de autobús frente a la edificación, ampliando el área de la acera en el punto donde sea el desembarque de los estudiantes. Sin embargo, en caso de escuelas urbanas, los puestos de estacionamientos podrán calcularse según la ordenanza vigente en la localidad (Gerencia de proyectos FEDE, 2007).

Cuando sea necesario pueden ubicarse también escaleras para las circulaciones verticales; las mismas deben cumplir con lo señalado por las normas FEDE, donde se indica que sus peldaños y escalones deben ser lisos, sin salientes y sin huecos, haciendo la precisión de que al finalizar el pasillo que comunique con la escalera o bien con la rampa, tienen que hacerse un cambio de textura de pisos para facilitar el tránsito de las personas invidentes. No es aconsejable utilizar escaleras de caracol, o escaleras con peldaños en los descansos ni en ángulo, así como las escaleras compensadas ya que en muchos de los casos, dichas escaleras servirán como vías de escape. La huella recomendada es de $30 \mathrm{~cm}$. y la contra huella de $15 \mathrm{~cm}$. Se debe evitar escaleras compensadas y de caracol.

Se propone también el uso de rampas cuando los niveles lo requieran, la misma tendrá una inclinación de $6 \%$, dependiendo del recorrido y la distancia que cubra, debe incluirse no solo en los accesos, sino en todos los espacios y dependencias escolares que componen la propuesta, ya que se recomienda garantizarse los principios de accesibilidad para personas con alguna discapacidad. Siguiendo lo indicado en la norma FEDE, las rampas de acceso, deberán tener dos pasamanos a diferentes alturas, el primer pasamano a 0.90 centímetros del nivel de piso acabado y el segundo a 0.70 centímetros del nivel de piso acabado.

Los pasillos de acceso a las aulas deben ser amplios y espaciosos, para facilitar la movilidad de los estudiantes, es recomendable evitar enfrentar módulos, que generen pasillos internos, ya que ello menoscaba la ventilación e iluminación natural de los mismos; cuando sean necesarios los módulos enfrentados, la norma FEDE prevé, pasillo con 3 metros de ancho, sin embargo siguiendo el criterio de modulación propuesto, la medida mínima deberá ser de 3,5 metros para, así facilitar el empalme estructural de los módulos.

Las puertas a las aulas, talleres y salones deberán abrir hacia afuera, evitando enfrentarse unas con otros; las dimensiones propuestas son las que indica la norma FEDE, con anchos de 0,90 metros hasta 1,20 metros, con doble hoja en el sentido más amplio; se mantiene además la altura mínima recomendada de 2,10 metros.

Las escuelas deberán tener relación con el contexto, privilegiando el aspecto funcional y espacial; para ello deberá preverse que ciertas áreas escolares puedan compartirse con la comunidad: los espacios deportivos, el aula de música, el taller de arte y el salón de usos múltiples. Se recomienda que los mismos puedan ser utilizados en horarios no escolares, sin embargo no se sugiere el uso de accesos auxiliares para estos, por lo que va en detrimento de la seguridad y el control que se tiene sobre el edificio escolar público. Ya que como señala Gutiérrez (2009) no debería existir un contraste enfatizado entre el edificio escolar y la arquitectura de la ciudad, en la que este se inserta.

En cuanto a la zonificación, es prudente mantener algunos de los aspectos señalados por la norma FEDE, como es el caso de la ubicación del edificio administrativo; el mismo debe contener los accesos escolares, así como también, estar ubicado en el nivel de planta baja; esta condición le denota como la fachada principal de la escuela, y por ende la que incide directamente sobre la vía urbana. Tómense en cuenta estos aspectos al momento del diseño, en lo referente a accesibilidad, señalización y presencia urbana del plantel educativo. Esta premisa se plantea en concordancia con lo indicado por Gutiérrez (2009), que entiende el edificio escolar en relación funcional y espacial al contexto en el que se emplaza. 
Se sugiere que la plaza de recreo y formación (o también llamada Plaza cívica), sea el elemento articulador del conjunto escolar; el mismo debe estar dotado del equipamiento necesario para su funcionamiento, como bancas, luminarias para los horarios vespertinos y nocturnos, áreas verdes con zonas de sombra, papeleras, y astas para banderas en zona visible y destacada; el acabado de piso deberá resistir el tránsito de muchas personas; opuesto a ella puede ubicarse la plataforma con un desnivel que funcione a modo de tarima para los eventos. Como señala Gutiérrez (2009), la escuela debe garantizar la movilidad dinámica de los estudiantes y la comunidad en general.

La norma FEDE, establece que tanto el cafetín, como el área deportiva, la plaza cívica y el área de usos múltiples deben estar en lo posible relacionados entre sí, por ser usos afines, estos además pueden ser utilizados por la comunidad en general. Las relaciones espaciales que se establezcan para estos menesteres no deberán perjudicar la actividad escolar, y por ende, se recomienda que se encuentren separados, mediante espacios articuladores: plazas, caminerías, áreas verdes, entre otras.

Debe tenerse especial cuidado con los espacios del pre-escolar; las aulas deben estar dotadas de patios y jardines internos e individuales, que permitan la salida y recreación controlada de los niños; cada aula debe poseer sus propios servicios sanitarios, su espacio de guardado para materiales didácticos y el mobiliario necesario para colocar las loncheras, termos y útiles escolares; la ubicación del pre-escolar debe ser la adecuada, para evitar conflictos con otros espacios que vinculen niños y usuarios de mayor edad.

Por norma FEDE, también se recomienda que las áreas de cocina, comedor, cuartos de servicio y cuarto de basura, estén directamente relacionadas con la vialidad exterior y de preferencia en los niveles de planta baja, por razones de uso y funcionamiento; para ello debe preverse un acceso de servicio, que facilite la entrada del personal de mantenimiento, la ubicación del tanque de aguas blancas y del tanque séptico (cuando sea necesario), así como también el paso de los camiones cisternas que sirven a dichos sistemas de almacenamiento; puede también vincularse el área de servicio a las zonas de estacionamientos.

Es recomendable dotar a las escuelas de un tanque de almacenamiento de aguas grises, que sirva para el regado de los jardines o bien de dispositivos de almacenamiento de las aguas de lluvia; claro está, dichos sistemas deben ser independientes a la dotación de aguas blancas y al drenaje de las aguas negras.

Es favorable mantener el criterio establecido por la norma FEDE de concentrar los núcleos sanitarios de varones, mujeres y profesores. Así como también utilizar la pared común o de servicio, para ahorro de las instalaciones y su mantenimiento. Se debe hacer hincapié, que es de carácter obligatorio, la ubicación de un cubículo para discapacitados dentro de cada núcleo sanitario, tanto para hembras como para varones.

En cuanto al paisajismo, se recomienda su utilización dentro del conjunto escolar, como indica la norma FEDE; debe aprovecharse este para la siembra de árboles o arbustos propios de la zona donde se realice el proyecto; es muy importante evitar el uso de árboles frutales, arbustos con espinas o de raíces superficiales, que puedan causar daños a los estudiantes, o bien que levanten porciones de piso en plazas y caminerías. Puede ser beneficioso también mantener las zonas de viveros, donde los mismos estudiantes puedan realizar prácticas de siembras de semillas, y reforzar la formación ecológica de los mismos.

También se recomienda mantener la zona de juegos pedagógicos, como un espacio abierto, vinculado a los patios, plazas y caminerías, solo que la propuesta de diseño podrá contener un lenguaje arquitectónico más consonó y actualizado, evitando reminiscencias ajenas a la localidad y a la cultura zuliana. De acuerdo con Gutiérrez (2009) la escuela debe expresar el modelo de educación impartido, por ello la propuesta deberá adaptarse al tiempo y la contemporaneidad del momento en el que se ejecuta. 
Portillo, R. - Criterios de diseño para las edificaciones educativas del estado Zulia, Venezuela.

En las escuelas bilingües, donde participe la comunidad indígena de la localidad, deberán preverse espacios para la transmisión de su propia cultura, espacios para tejidos, formación lingüística, y aspectos folclóricos. En dicha propuesta deben prevalecer los espacios abiertos en relación con la naturaleza, según los criterios propios de los estilos de vida de dichas comunidades; para su programación debe consultarse a los usuarios específicos.

En cuanto a la forma, según la norma FEDE, deben evitarse superficies cóncavas reflectoras que concentren y focalicen la energía sonora y causen ecos en el complejo escolar; para las actividades académicas en muchos casos es necesario silencio y concentración, por lo cual las formas utilizadas deben remarcar dichos aspectos. Deben evitarse además las superficies rígidas, paralelas y pulidas que produzcan fenómenos de reflexión.

El lenguaje formal deberá lograrse de manera creativa, siguiendo los esquemas planteados por FUNDAEDUCA, o bien añadiendo nuevas maneras de agrupación de espacios; debe concebirse la propuesta arquitectónica como una unidad formal y funcional, evitando disgregar módulos a modo de islas, sino unificando como un todo la edificación; este criterio responde también a aspectos de seguridad y control sobre los mismos espacios. Se recomiendan dos niveles de altura como máximo.

Se sugiere además que las formas utilizadas posean un lenguaje consonó y actualizado, evitando reminiscencias a estilos arquitectónicos ajenos a la contemporaneidad cultural de la región. La forma debe expresar la importancia que tiene el plantel en la comunidad y en la sociedad, y el papel protagónico que tiene la educación como proceso social de transmisión de valores. De allí que los conceptos de diseño que se propongan, sean cónsonos con el modelo educativo y pedagógico que se imparta en dichos espacios.

Se recomienda mantener la norma FEDE, donde indica que la altura mínima de los espacios docentes sea de 3,00 metros, considerando dicha dimensión desde el piso acabado, al punto más bajo del techo (suponiendo que lleva alguna inclinación). Al respecto hay que añadir que por razones ambientales, se recomiendan techos con ángulos inclinados, así como también para facilitar el mantenimiento de la cubierta y el drenaje de las aguas de lluvia. Los techos planos podrían ser considerados en el diseño, para proyectar futuras ampliaciones en niveles superiores, habiendo recibido el tratamiento adecuado para ello. Sin embargo queda a criterio del equipo de diseño resolver acertadamente el tema de las cubiertas.

Desde el punto de vista, de la ventilación natural, deberán tomarse en cuenta las recomendaciones aportadas por la norma FEDE; en cuanto a la ubicación de áreas verdes en las zonas aledañas de los espacios, "la colocación correcta de vegetación permite la absorción de radiación solar y enfriamiento del aire que penetra en la edificación entre otros dispositivos y soluciones" (Gerencia de proyectos FEDE, 2007. P. 102). En las escuelas públicas por razones económicas y de seguridad, se mantiene el principio de ventilación natural, reforzada por dispositivos mecánicos como ventiladores de techo; sin embargo es muy importante que la ubicación de los mismos no constituya un peligro para los estudiantes, de ser esta opción de diseño la acordada, deberá tomarse en cuenta al momento de definir las alturas de los espacios, manteniendo el criterio anterior de 3,00 metros como mínimo de planta libre.

Para la utilización de elementos en las fachadas, como quiebra soles, persianas, pantallas, romanillas, aleros, y altos relieves, deberá tomarse en cuenta los efectos ambientales que los mismos generan en el interior de los espacios del complejo escolar; estos deben facilitar el recorrido de los vientos y no ser un obstáculo para los mismos, tal como lo indica la norma FEDE, que asegura que los elementos de fachadas deben retirarse al menos 30 centímetros del plano que cubren. Sin embargo para un mejor funcionamiento a modo de dar mantenimiento a dichos elementos, el retiro mínimo óptimo que se propone, debería oscilar entre los 60 y 70 centímetros del plano liso de la fachada, donde se garantice

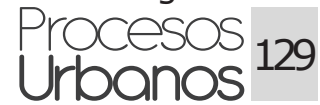


el paso de una persona; mientras que para los aleros, el ancho mínimo óptimo propuesto sería de 1,20 metros.
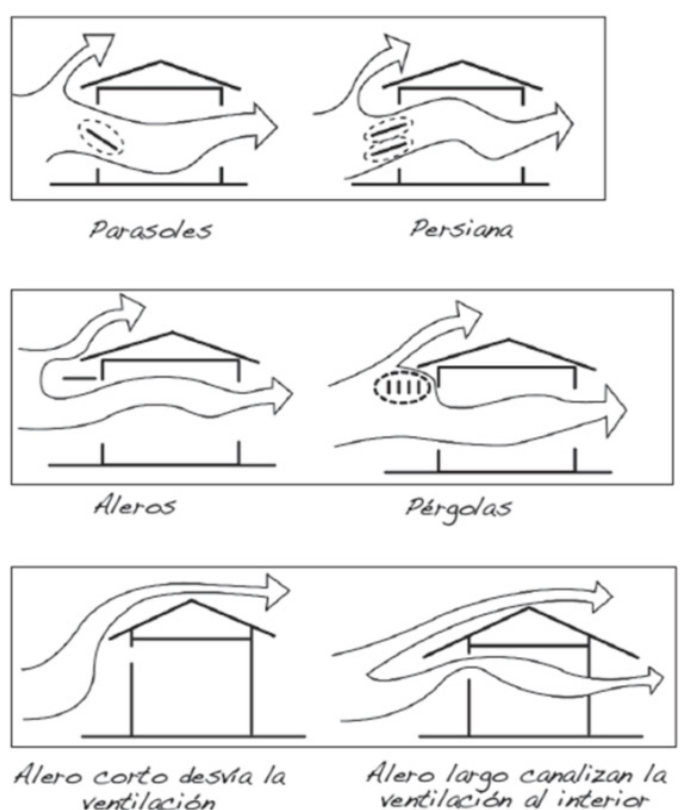

Figura 1. Elementos de fachada y la ventilación. Fuente: Gerencia de proyectos FEDE (2007).

En cuanto a las ventanas deberá privilegiarse el paso de la luz natural, tomando siempre como referencia para su ubicación las caras laterales de las aulas, y nunca las fachadas donde se encuentre el pizarrón, ni la cara posterior; deberán evitarse además las ventanas fragmentadas, privilegiándose los planos abiertos horizontales sobre los verticales; al respecto, la norma FEDE indica que ningún antepecho podrá ser menor a 1 metro, mientras que en las aulas con vistas internas hacia pasillos u otros módulos, se recomienda que el antepecho no sea menor a 1,60 metros; sin embargo, por medidas de seguridad ningún antepecho podrá ser mayor a 2,10 metros, considerándose este como el máximo para zonas sanitarias y de servicios.

Las ventanas podrán ser de cualquier tipo, siempre y cuando se garantice el paso de la luz y la ventilación natural; deben evitarse sistemas complicados y difíciles de mantener económicamente y que no aporten seguridad; en cuanto a los vidrios deben ser transparentes y traslúcidos, no se permiten vidrios, escarchados, esmerilados, ni recubiertos por ningún tipo de papel; esto se debe también a razones de funcionalidad y seguridad. Debe garantizarse por tanto la mayor transparencia e iluminación natural posible.

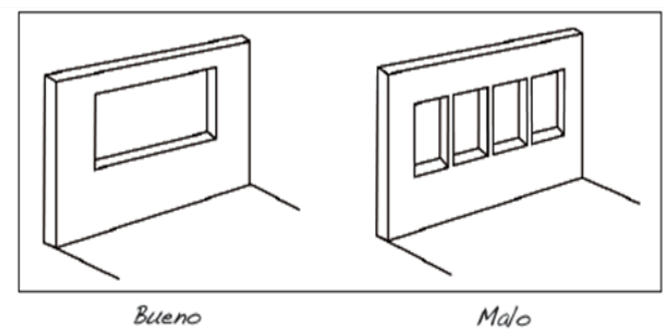

Figura 2. Aberturas recomendadas para la iluminación. Fuente: Gerencia de proyectos FEDE (2007).

En cuanto al acabado de los pisos, tanto interiores como exteriores, deberán ser de materiales anti resbalantes y de alta durabilidad, que no impliquen costos elevados para su mantenimiento, ni dificultad para su limpieza; serán resistentes e impermeables; evítense pisos brillantes, como cerámicas, caicos, o piedras naturales pulidas; para los pisos exteriores debe garantizarse el drenaje de las aguas de lluvia, de modo que no se acumulen; se recomiendan pisos rústicos, texturizados y resistentes.

Para los sistemas constructivos, la idea es que puedan garantizar una rápida ejecución de la obra, sin menoscabar la calidad de la misma. Aunque el sistema tradicional es el mayormente utilizado en la actualidad, pueden considerarse algunos elementos pre-fabricados para garantizar la agilidad constructiva que se requiera y no demorar en años la construcción de la escuela.

Para el acabado de la cubierta no es recomendable el uso de tejas criollas, ya que son susceptibles a roturas, y solo aportan un carácter ornamental a la propuesta arquitectónica; para ello pueden emplearse acabados en techos, con mayor resistencia, durabilidad y un mínimo de mantenimiento, que garanticen el drenaje de las aguas de lluvia.

Se sugiere que los techos internamente sean de color blanco, por el tema de la iluminación; el acabado será pintura mate, acrovinílica; deben evitarse las pinturas a base de aceites 
Portillo, R. - Criterios de diseño para las edificaciones educativas del estado Zulia, Venezuela.

y reflectantes en las superficies de techo, ya que perjudican el efecto visual que se requiere en el aula de clases.

Las paredes también deben ser recubiertas con materiales resistentes; se recomienda la pintura mate acrovinílica, sobre el encamisado, con superficie lisa; no están permitidos en los espacios internos los acabados texturizados; se recomienda no usar pintura a base de aceites o reflectantes en las aulas; en el caso que sea necesario, por razones de mantenimiento, la misma tendrá una altura de 1,00 metro, y el resto de la superficie sea recubierta con pintura mate.

Para los baños, talleres y laboratorios, se recomienda el uso de cerámicas y superficies de fácil limpieza, sin texturas, con superficie lisa, que garantice la no adherencia de cualquier sustancia; las esquinas de los espacios y de los topes deben ser redondeadas, por medidas de seguridad.

En cuanto a los colores, se recomienda el uso de colores fríos y neutrales, con preferencia de color blanco; los mismos según norma FEDE, producen sensación de reposo y deben ser utilizados en ambientes bien iluminados con muchas ventanas, son perfectos para dar sensación de frescura y amplitud. Los colores cálidos, solo están permitidos por la norma, para los espacios exteriores, siempre y cuando se corresponda con el uso acorde de los mismos para representar las actividades escolares; en ningún caso debe usarse el color para manifestar tendencias político partidistas, ya que la educación es un proceso social, pluralista, sin fines de adoctrinamiento, sino con el objetivo de la formación integral de la persona humana, en beneficio de la sociedad.

\section{CONSIDERACIONES FINALES.}

Los criterios de diseño, planteados relacionados con las normas FEDE, son pautas no rígidas, ni estáticas, que buscan motivar la reflexión y el pensamiento creativo del equipo de diseño. En muchos de los casos, el estudio ha optimizado la escueta especificación de la normativa nacional, y se han regionalizado, a modo de que las propuestas de diseño que se generen de estas, no sean una repetición en serie, sino la búsqueda por satisfacer acertadamente las necesidades espaciales de las comunidades que requieran de espacios escolares, en los niveles de pre-escolar y básica.

Los criterios de diseño de las escuelas zulianas deben ser un continuo plan de trabajo, ellos no son excluyentes, requieren estar abiertos a la innovación, a la creatividad, a la reflexión y a la motivación para incluir nuevos y mejores elementos, de tal manera que la lista propuesta puede irse ampliando, revisando y actualizando con base en los cambios que demande la sociedad; la educación tiene un lugar privilegiado en el proceso de desarrollo y de progreso de cualquier comunidad o país; los criterios de diseño necesitan responder a este principio, deben ser respuestas que indiquen el camino hacia mejoras en la concepción, formulación, diseño y ejecución de los edificios escolares, donde se forja el futuro de las generaciones. Se recomienda también que los organismos públicos de la región zuliana revisen los criterios de diseño aplicados en la actualidad, considerando otros elementos innovadores como los generados en esta investigación. Aunque el estudio solo consideró las escuelas financiadas con inversión pública, como resultante del proceso de gestión de gobierno regional, puede promoverse la participación de la empresa privada, para que mediante programas de responsabilidad civil, también pueda involucrarse para el financiamiento de dichas obras, adjudicando recursos directamente con la comunidad servida.

Debe también motivarse en las comunidades el conocimiento sobre los estándares de calidad y criterios de diseño necesarios para la construcción de nuevas unidades escolares, a fin de que puedan demandar mejoras en cuanto al tema.

Este estudio corresponde a resultados parciales de investigación titulada "Criterios generales de diseño para las edificaciones educativas del estado Zulia". 


\section{REFERENCIAS.}

Asamblea nacional de la República Bolivariana de Venezuela (2009), Ley orgánica de educación, Caracas Venezuela, Palacio Federal Legislativo, Gaceta Oficial no extraordinario 5.929 de fecha 15 de agosto de 2009.

FEDE, Fundación de Edificaciones y Dotaciones Educativas (2015). Quiénes somos. http:// www.fede.gob.ve/web/index.php/la-fundacion/quienes-somos [Consulta: 8 de abril de 2015].

Fonseca, X. (1994). Las medidas de una casa, antropometría de la vivienda, primera edición, ciudad de México, México, Editorial Pax. pp. 9.

Gerencia de proyectos de FEDE. Fundación de Edificaciones y Dotaciones Educativas (2007). Normas y recomendaciones para el diseño de edificaciones educativas. Caracas, Venezuela. Editado por el Ministerio del Poder Popular la Educación, pp. 29-230.

Gobernación del estado Zulia (2002), Decreto para la creación de la Fundación para la Infraestructura de la Planta Física Educativa del estado Zulia (FUNDAEDUCA). Decreto Gubernamental No 402, de fecha 06 de noviembre de 2002, Gaceta Oficial Extraordinaria del estado Zulia no 735 de fecha 30 de noviembre de 2002.

Gutiérrez Paz, J. (2009). Estándares básicos para construcciones escolares, una mirada crítica. Revista Educación y Pedagogía. Volumen 21, número 54 mayo-agosto, pp 155-176.

López, O.; Marinilli, Á., Bonilla, R., Fernández, N., Domínguez, J., Coronel, G.; Baloa, T.; Vielma, R. (2010). Evaluación sismorresistente de edificios escolares en Venezuela. Revista Facultad de Ingeniería UCV. Volumen 25, número 4, pp 81-94.

Loyola Vergara, M.; Goldsack L. (2010). Constructividad y Arquitectura, Santiago de Chile, Chile, primera edición, Editorial de la Universidad de Chile, pp. 69-70.

Ministerio para el Poder Popular de la Educación (2014). "Gobierno nacional apuesta a la excelencia educativa". http://www.me.gob.ve/noticia.php?id contenido=28212 . [Consultado: 20 de abril de 2015].

Rapoport, A. (2003). Cultura, arquitectura y diseño, volumen 5 de Arquitectonics. Mind, land \& society, primera edición. Barcelona, España, Editorial de la Universidad Politécnica de Catalunya, pp. 114-115.

Sanoff, H. (2006). Programación y participación en el diseño arquitectónico, volumen 12 de Arquitectonics: Mind, land \& society, primera edición. Barcelona, España, Editorial de la Universidad Politécnica de Catalunya, pp. 116.

UNESCO (2015). Objetivos del milenio. http://www.unesco.org/new/es/our-priorities/ education-for-all/ . [Consultado: 20 de abril de 2015].

Instituto Nacional de Estadísticas. INE. (2015). Matrícula de educación primaria por dependencia, según año escolar, 2003/04-2012/13 Disponible en: http://www.ine.gov.ve/ index. php?option $=$ com content\&view $=$ category\&id=64\&Itemid=39\# . [Consultado: 18 de abril de 2015]. 